\title{
Performance of GMRES for the MFS
}

\author{
A. Karageorghis \& Y.-S. Smyrlis \\ Department of Mathematics and Statistics, University of Cyprus, Cyprus
}

\begin{abstract}
In this work we present some preliminary numerical results regarding the performance of the Generalized Minimal Residual (GMRES) method when it is applied to the solution of the linear systems arising from the discretization of certain elliptic boundary value problems in two and three dimensions by the Method of Fundamental Solutions (MFS).

Keywords: method of fundamental solutions, generalized minimal residual method, Laplace equation, iterative methods.
\end{abstract}

\section{The problem and method}

We consider the solution of Laplace's equation

$$
\Delta u=0 \text { in } \Omega
$$

subject to the Dirichlet boundary condition

$$
u=f \quad \text { on } \quad \partial \Omega
$$

where the domain $\Omega$ is bounded in $\mathbb{R}^{d}, d=1,2$ and $f$ is a given function.

In the MFS [1], the solution $u$ of (1.1)-(1.2) is approximated by

$$
u_{N}(\boldsymbol{c}, \boldsymbol{Q} ; P)=\sum_{\ell=1}^{N} c_{\ell} K_{d}\left(P, Q_{\ell}\right), \quad P \in \bar{\Omega},
$$

where $\boldsymbol{c}=\left(c_{1}, c_{2}, \ldots, c_{N}\right)^{T}$ and $\boldsymbol{Q}$ is a $d N$-vector containing the coordinates of the singularities $Q_{\ell}, \ell=1, \ldots, N$, which lie outside $\bar{\Omega}$. The function $K_{d}(P, Q)$ is a fundamental solution of Laplace's equation given by 


$$
K_{d}(P, Q)=\left\{\begin{array}{cc}
-\frac{1}{2 \pi} \log |P-Q|, & d=2, \\
\frac{1}{4 \pi} \frac{1}{|P-Q|}, & d=3,
\end{array}\right.
$$

with $|P-Q|$ denoting the distance between the points $P$ and $Q$. As recommended in [2], the singularities $Q_{\ell}$ are fixed on a pseudo-boundary $\partial \tilde{\Omega}$ similar to $\partial \Omega$ and a set of collocation points $\left\{P_{k}\right\}_{k=1}^{N}$ is placed on $\partial \Omega$.

The coefficients $c$ are determined so that the boundary condition is satisfied at the boundary points $\left\{P_{k}\right\}_{k=1}^{N}$ :

$$
u_{N}\left(\boldsymbol{c}, \boldsymbol{Q} ; P_{k}\right)=f\left(P_{k}\right), \quad k=1, \ldots, N .
$$

This yields a linear system of the form

$$
G^{d} c=f
$$

for the coefficients $c$, where the elements of the matrix $G^{d}$ are given by

$$
G_{k, \ell}^{d}=\left\{\begin{aligned}
-\frac{1}{2 \pi} \log \left|P_{k}-Q_{\ell}\right|, & d=2, \\
\frac{1}{4 \pi} \frac{1}{\left|P_{k}-Q_{\ell}\right|}, & d=3,
\end{aligned}\right.
$$

for $k=1, \ldots, N \quad \ell=1, \ldots, N$.

The matrix $G^{d}$ is full and, in general, not symmetric. Also, as the pseudoboundary $\partial \tilde{\Omega}$ moves away from the boundary $\partial \Omega$ the matrix becomes very illconditioned resulting in loss of accuracy of the MFS approximation [3].

In most applications of the MFS so far, system (1.6) is solved using standard Gaussian elimination at a cost of $O\left(N^{3}\right)$ operations. The question is whether an iterative technique would lead to savings in the cost of solving the system and the effect it could have on the accuracy of the approximation. Since the matrix $G^{d}$ is full and, in general, not symmetric, and as recommended in the literature (see, for example [4]) we shall experiment with the Generalized Minimal Residual (GMRES) method derived by Saad and Schultz [5].

In this work we shall try and address the following two questions:

1. When $N$ is large the cost of solving (1.6) is obviously large. Would the cost be reduced if one used an iterative method to solve system (1.6)? In order to address this question we shall investigate the performance of GMRES.

2. When the distance of the pseudo-boundary from the boundary is large there is the additional problem that the matrix $G$ in (1.6) becomes ill-conditioned. Would the use of a pre-conditioner in GMRES improve the situation [6]? 
What would a suitable choice for such a pre-conditioner be? We shall experiment by taking the pre-conditioner $P$ to be a diagonal matrix consisting of the diagonal elements of $G^{d}$. Such pre-conditioners were found to be effective when using GMRES to solve systems resulting from Boundary Element Method (BEM) discretizations [7-9].

\section{Well-conditioned systems}

We first consider the case when the MFS systems are (relatively) well-conditioned. This occurs when the distance of the pseudo-boundary from the boundary is small, and becomes smaller with increasing $N$.

\subsection{Example 1}

We consider the boundary value problem (1.1)-(1.2) when $\Omega \subset \mathbb{R}^{2}$, in particular when $\Omega$ is the square $(-1,1) \times(-1,1)$ and $f$ corresponds to the exact solution $u(x, y)=\mathrm{e}^{x} \cos y$. The boundary points and the singularities are uniformly distributed around the boundary and pseudo-boundary, respectively. We calculated the maximum error on a set of uniformly distributed points on the boundary (different from the boundary collocation points) for various values of the distance of the pseudo-boundary from the boundary, ranging from 0 to 0.05 . In Figures 1(a), 1(b), 1(c) and 1(d), in the upper subplots we present the maximum error on the boundary, obtained (i) with Gaussian elimination, (ii) with $4 \log _{2} N, 8 \log _{2} N$ and $12 \log _{2} N$, iterations of GMRES, and (iii) preconditioned GMRES, in each case, versus the distance of the pseudo-boundary from the boundary, for $N=400,600,800$ and 1000 , respectively. In the lower subplots of each figure we present the 2-norm condition number $\kappa_{2}$ of the matrix $G$ versus the distance of the pseudo-boundary from the boundary. The results indicate that for values of $\kappa_{2}$ less than $10^{20}$, the results obtained with GMRES are indistinguishable from those obtained with Gaussian elimination for as few as $4 \log _{2} N$ GMRES iterations. When $\kappa_{2}$ reaches $10^{20}$, $4 \log _{2} N$ GMRES were no longer sufficient. The number of GMRES iterations started depending on both the distance from the boundary and the value of $N$. For $N=400,4 \log _{2} N$ iterations yielded better results than Gaussian elimination for a distance up to 0.04 , whereas $8 \log _{2} N$ and $12 \log _{2} N$ iterations yielded better results than Gaussian elimination for a distance up to 0.05 . For $N=600$, $4 \log _{2} N$ iterations yielded better results than Gaussian elimination for a distance up to 0.02 , whereas $8 \log _{2} N$ and $12 \log _{2} N$ iterations yielded better results than Gaussian elimination for a distance up to 0.05 . For $N=800,4 \log _{2} N$ iterations yielded better results than Gaussian elimination for a distance up to 0.01 , $8 \log _{2} N$ iterations yielded better results than Gaussian elimination for a distance up to 0.022 , whereas $12 \log _{2} \mathrm{~N}$ iterations yielded better results than Gaussian elimination for a distance up to 0.032 . The difference in accuracy between GMRES and Gaussian elimination for $8 \log _{2} N$ and $12 \log _{2} N$ iterations was small all the way up to 0.05 . Finally, for $N=1000,4 \log _{2} N$ iterations did not yield better results than Gaussian elimination beyond the point where $\kappa_{2}$ reaches $10^{20}, 8 \log _{2} N$ iterations yielded better results than Gaussian elimination for a distance up to 0.018 , 


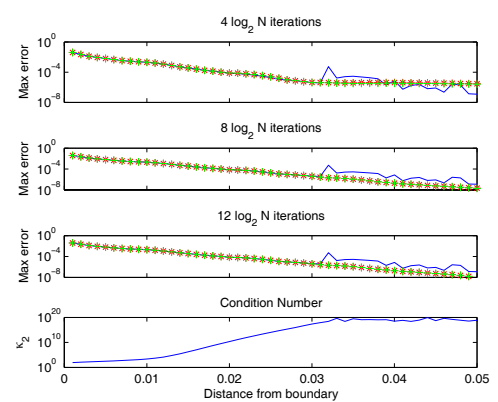

(a) $N=400$
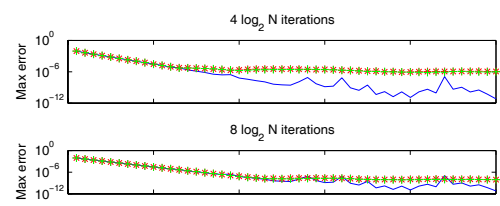

$12 \log _{2} N$ iterations
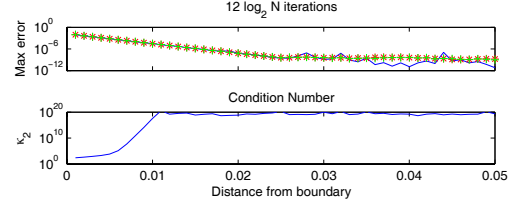

(c) $N=800$

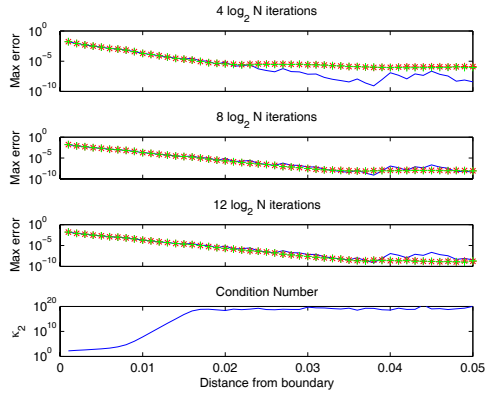

(b) $N=600$

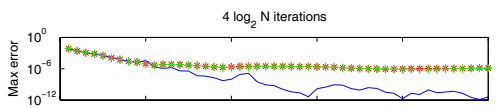

$8 \log _{2} N$ iterations
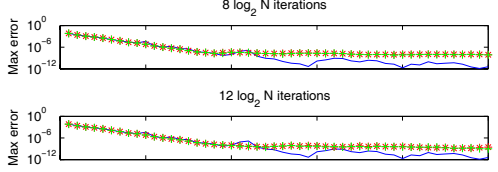

Condition Number

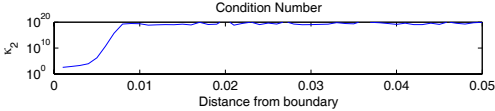

(d) $N=1000$

Figure 1: Example 1: Plots with $\ell \log _{2} N$ GMRES iterations $\ell=4,8,12$. Key: - Gaussian elimination, * GMRES, + preconditioned GMRES.

whereas $12 \log _{2} N$ iterations yielded better results than Gaussian elimination for a distance up to 0.024. The difference in accuracy between GMRES and Gaussian elimination for $8 \log _{2} \mathrm{~N}$ and, in particular, $12 \log _{2} \mathrm{~N}$ iterations was small all the way up to 0.05 . No particular improvement was observed for the pre-conditioned GMRES. In Figure 2 we present the timings recorded using the Matlab commands tic and toc for various values of $N$ and iterations and using Gaussian elimination. We observe that with the exception of $12 \log _{2} N$ iterations, in the other cases substantial savings are achieved using GMRES. Note that for GMRES we used the Matlab command gmres with the tolerance set at TOL $=1 . e-10$.

\subsection{Example 2}

We consider the boundary value problem (1.1)-(1.2) when $\Omega \subset \mathbb{R}^{3}$, in particular when $\Omega$ is the cube $(-1,1) \times(-1,1) \times(-1,1)$ and $f$ corresponds to the exact solution $u(x, y)=\cosh (0.3 x) \cosh (0.4 y) \cos (0.5 y)$. The boundary points and the singularities are uniformly distributed around the boundary and pseudoboundary, respectively. As in Example 1, we calculated the maximum error on a 


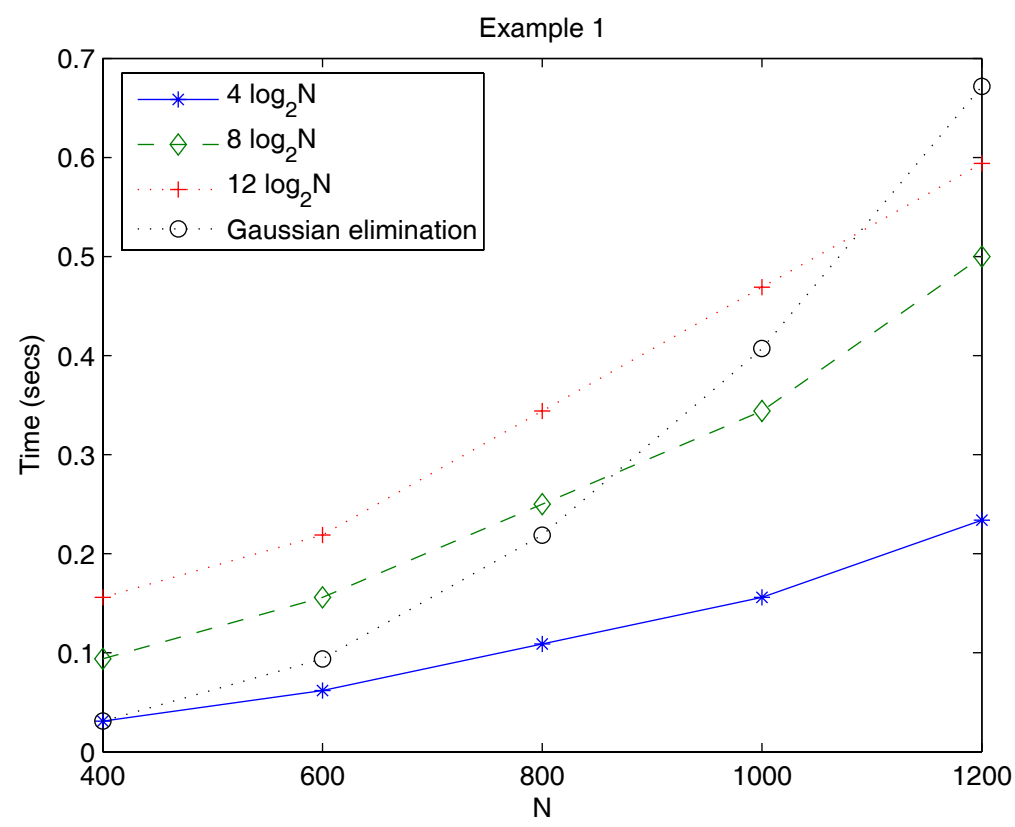

Figure 2: Example 1: Timings recorded for various numbers of degrees of freedom and iterations.

set of uniformly distributed points on the boundary (different from the boundary collocation points) for various values of the distance of the pseudo-boundary from the boundary. In Figures 3(a), 3(b) and 3(c), in the upper subplots we present the maximum error on the boundary, obtained (i) with Gaussian elimination, (ii) with $4 \log _{2} N, 8 \log _{2} N$ and $12 \log _{2} N$, iterations of GMRES, and (iii) preconditioned GMRES, in each case, versus the distance of the pseudo-boundary from the boundary, for $N=600,864$ and 1176 (i.e. $6 \times 10^{2}, 6 \times 12^{2}$ and $6 \times 14^{2}$ ), respectively. In the lower subplots of each figure we present the 2-norm condition number $\kappa_{2}$ of the matrix $G$ versus the distance of the pseudo-boundary from the boundary. The observations from these plots are similar to the ones drawn from the corresponding plots for Example 1. Interestingly, the conditioning of the MFS matrix for the three-dimensional example is considerably better that the one for the twodimensional example, in the sense that the condition number $\kappa_{2}$ stays below $10^{20}$ for considerably longer distances of the pseudo-boundary from the boundary. As a result, the range of distances of the pseudo-boundary to the boundary was extended from 0 up to 2 . For $N=600,4 \log _{2} N$ iterations produced identical results to Gaussian elimination for a distance up to 1 , while remaining very close up to 2 . For both $8 \log _{2} N$ and $12 \log _{2} N$ iterations the GMRES results were identical to the results obtained with Gaussian elimination. For $N=864,4 \log _{2} N$ iterations 

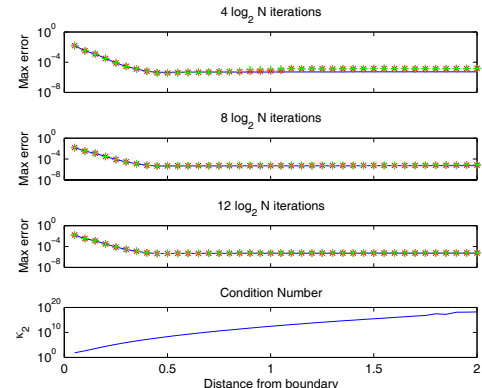

(a) $N=600$

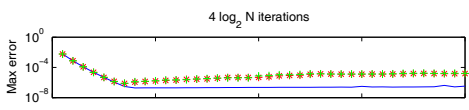

$8 \log _{2} N$ iterations
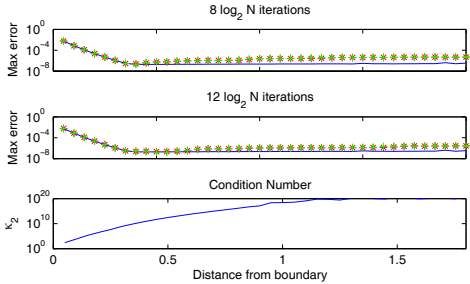

(c) $N=1176$
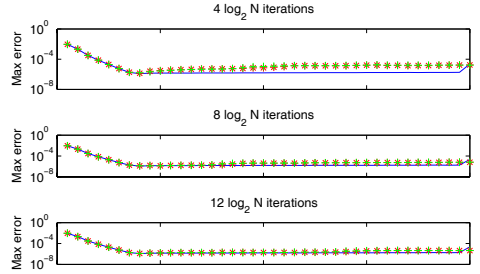

Condition Number

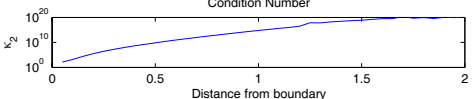

(b) $N=864$

Figure 3: Example 2: Plots with $\ell \log _{2} N$ GMRES iterations $\ell=4,8,12$. Key: - Gaussian elimination, * GMRES, + preconditioned GMRES.

yielded identical results to Gaussian elimination for a distance up to 0.5 , while remaining close up to 2 . For $8 \log _{2} N$ iterations the GMRES results were identical to those obtained with Gaussian elimination up to 0.8 and for $12 \log _{2} N$ iterations this range went up to 1.5 . For both $8 \log _{2} N$ and $12 \log _{2} N$ iterations the GMRES results were very close to the results obtained with Gaussian elimination up to 2 . For $N=1176,4 \log _{2} N$ iterations yielded identical results to Gaussian elimination for a distance up to 0.25 . For $8 \log _{2} N$ iterations the GMRES results were identical to those obtained with Gaussian elimination up to 0.5 and for $12 \log _{2} \mathrm{~N}$ iterations this range went up to 0.7 . For both $8 \log _{2} N$ and, in particular, $12 \log _{2} N$ iterations the GMRES results were very close to the results obtained with Gaussian elimination up to 2 .

\section{Ill-conditioned systems}

We now consider the case when the MFS systems are ill-conditioned. This occurs when the distance of the pseudo-boundary from the boundary is large, and becomes the ill-conditioning becomes more severe with increasing $N$. 


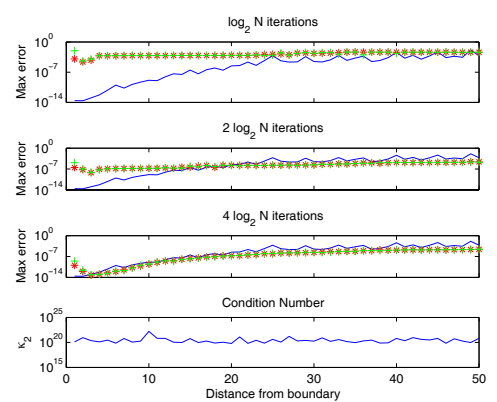

(a) $N=400$
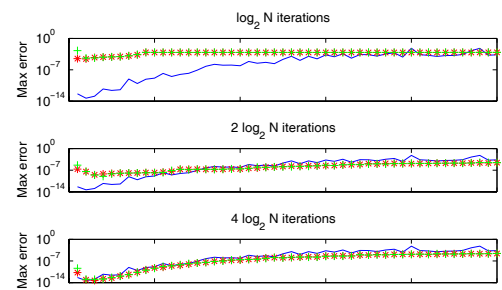

Condition Number

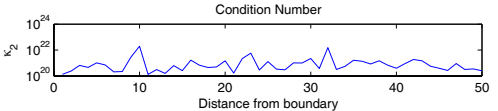

(c) $N=800$

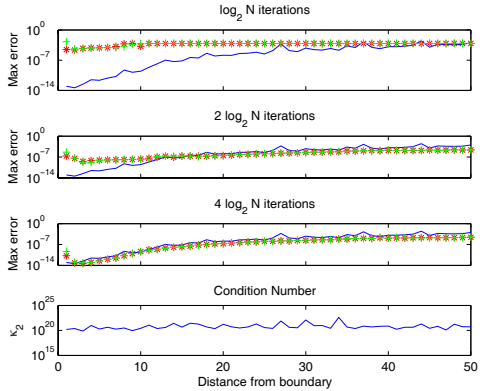

(b) $N=600$
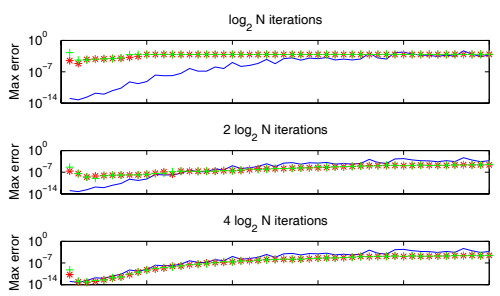

Condition Number

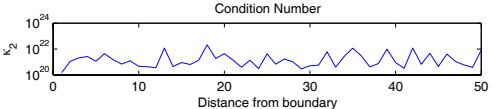

(d) $N=1000$

Figure 4: Example 1: Plots with $\ell \log _{2} N$ GMRES iterations $\ell=1,2,4$. Key: - Gaussian elimination, * GMRES, + preconditioned GMRES.

\subsection{Example 1}

As in Section 2.1 we calculated the maximum error on a set of uniformly distributed points on the boundary (different from the boundary collocation points) for various values of the distance of the pseudo-boundary from the boundary. This time we considered the range from 0 to 50. In Figures 4(a), 4(b), 4(c) and 4(d), in the upper subplots we present the maximum error on the boundary, obtained (i) with Gaussian elimination, (ii) with $\log _{2} N, 2 \log _{2} N$ and $4 \log _{2} N$, iterations of GMRES, and (iii) preconditioned GMRES, in each case, versus the distance of the pseudo-boundary from the boundary, for $N=400,600,800$ and 1000 , respectively. In the lower subplots of each figure we present the 2-norm condition number $\kappa_{2}$ of the matrix $G$ versus the distance of the pseudo-boundary from the boundary. The results indicate that in all cases, as few as $4 \log _{2} N$ GMRES iterations yield considerably more accurate results than Gaussian elimination, with the exception of the cases when the pseudo-boundary is very close to the boundary. No improvement was observed for more than $4 \log _{2} N$ GMRES iterations. Also, as in Section 2.1, no particular improvement was observed for the pre-conditioned GMRES. 


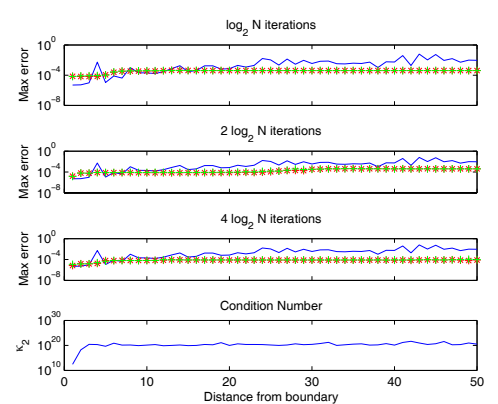

(a) $N=600$
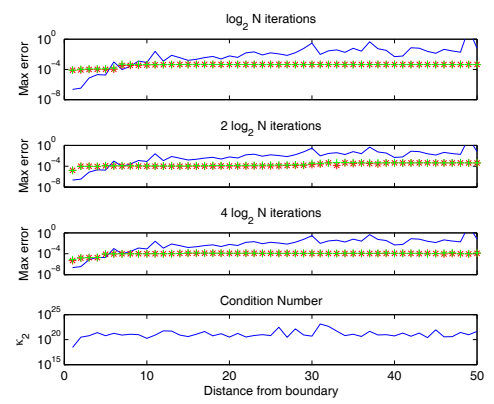

(c) $N=1176$
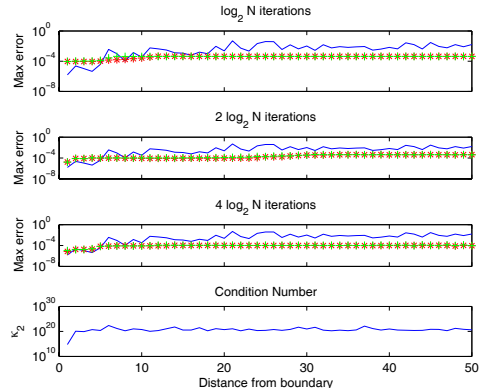

(b) $N=864$

Figure 5: Example 2: Plots with $\ell \log _{2} N$ GMRES iterations $\ell=1,2,4$. Key: - Gaussian elimination, $*$ GMRES, + preconditioned GMRES.

\subsection{Example 2}

We consider the example of Section 2.2 examining the maximum error for the distance of the pseudo-boundary from the boundary ranging from 0 to 50. In Figures 5(a), 5(b) and 5(c), in the upper subplots we present, as before, the maximum error on the boundary, obtained (i) with Gaussian elimination, (ii) with $\log _{2} N, 2 \log _{2} \mathrm{~N}$ and $4 \log _{2} N$, iterations of GMRES, and (iii) preconditioned GMRES, in each case, versus the distance of the pseudo-boundary from the boundary, for $N=600,864$ and 1176 , respectively. In the lower subplots of each figure we present the 2norm condition number $\kappa_{2}$ of the matrix $G$ versus the distance of the pseudoboundary from the boundary. The observations from these plots are similar to the ones drawn from the corresponding plots for Example 1, namely that for as few as $4 \log _{2} N$, GMRES iterations considerably more accurate results are obtained than using Gaussian elimination. No further improvement was observed for larger values of the number of iterations. 


\section{Conclusions}

In this work we carried out some preliminary numerical tests on the performance of GMRES when applied to the solution of the linear systems arising from the MFS discretization of certain two- and three-dimensional elliptic boundary value problems. For the case when the pseudo-boundary is located very close to the boundary, yielding well-conditioned systems, the accuracy of the iterative solver is satisfactory, although, in general inferior to that of Gaussian elimination. Interestingly, in the case when the pseudo-boundary is located far from the boundary, yielding ill-conditioned systems, the accuracy of the iterative solver is superior to that of Gaussian elimination, even for relatively few iterations. No improvement was observed in either case by the use of a preconditioned GMRES.

\section{References}

[1] Fairweather, G. \& Karageorghis, A., The method of fundamental solutions for elliptic boundary value problems. Numerical treatment of boundary integral equations. Adv Comput Math, 9(1-2), pp. 69-95, 1998.

[2] Gorzelańczyk, P. \& Kołodziej, J.A., Some remarks concerning the shape of the source contour with application of the method of fundamental solutions to elastic torsion of prismatic rods. Engng Analysis with Boundary Elements, 37, pp. 64-75, 2008.

[3] Smyrlis, Y.S. \& Karageorghis, A., Some aspects of the method of fundamental solutions for certain harmonic problems. J Sci Comput, 16(3), pp. 341-371, 2001.

[4] Demmel, J.W., Applied Numerical Linear Algebra. SIAM: Philadelhia, 1997.

[5] Saad, Y. \& Schultz, M.H., GMRES: A generalized minimal residual algorithm for solving non-symmetric linear systems. SIAM J Sci Statist Comput, 7, pp. $856-869,1986$.

[6] Greenbaum, A., Iterative Methods for Solving Linear Systems. SIAM: Philadelhia, 1997.

[7] Barra, L.P.S., Coutinho, A.L.G.A., Mansur, W.J. \& Telles, J.C.F., Iterative solution of BEM equations by GMRES algorithm. Comput \& Structures, 44, pp. 1249-1253, 1992.

[8] Guru Prasad, K., Kane, J., Keyes, D.E. \& Balakrishna, C., Preconditioned Krylov solvers for BEA. Internat J Numer Methods Engrg, 37, pp. 1651-1672, 1994.

[9] Marburg, S. \& Schneider, S., Performance of iterative solvers for acoustic problems. Part I. Solvers and effect of diagonal preconditioning. Engrg Anal Boundary Elem, 27, pp. 727-750, 2003. 